\title{
Implementation of BER Performance in SFBC/STBC Based On QAM-OFDM Detection Techniques in OFDM Systems
}

\author{
Ritesh Saraswat ${ }^{1}$, Vishwajeet Mishra ${ }^{2}$, Piyush Vyas ${ }^{3}$, Prof. K. K. Arora ${ }^{4}$ \\ Associate Professor, Department of ECE, JIET Group of Institutions, Jodhpur, Rajasthan, India \\ Assistant Professor, Department of ECE, JIET Group of Institutions, Jodhpur, Rajasthan, India \\ Assistant Professor, Department of ECE, JIET Group of Institutions, Jodhpur, Rajasthan, India \\ Associate Professor, Department of ECE, JIET Group of Institutions, Jodhpur, Rajasthan, India
}

\begin{abstract}
Cooperative wireless communication relay networks in OFDM-based systems have been shown to improve performance in various systems using digital modulation techniques as a form of spatial diversity, this method indeed improves the performance in comparison in various aspects of BER and SNR with fixed and random values of input applied on functions on FFT, IFFT, mixing of each other etc. to existing when filtering is necessary for band limited conditions. In this proposed paper, we are implementing the better and updation in results with the mathematical calculation in FFT, IFFT, and mixing of algorithms and terminology interfacing over the range of 256 and 512 - ary QAM OFDM techniques which are useful in communication scenarios. Scattering and conjugate plots are added for 256 and 512ary are implemented for orthogonal transmission, overlapping, narrow band sub channel or sub-carriers, transmitted in parallel data over transmission bandwidth.
\end{abstract}

KEYWORDS: OFDMA, Digital Techniques, M-ary QAM, cooperative diversity, best relay selection FFT, IFFT, SNR BER and their performance.

\section{INTRODUCTION}

Orthogonal Frequency Division Multiplexing (OFDM) is a digital modulation technique who consists of transmitting a data stream using a large number of parallel narrow-band sub carriers instead of a single wide-band carrier i.e. in OFDM multiplexing is applied to independent signals but these independent signals are a subset of one main signal. Therefore OFDM is a combination of modulation and multiplexing with better immunity to impulse noise and intersymbol interference (ISI), eye patterns in low complexity and high spectral efficiency. Implementation of OFDM modulation is illustrated by the OFDM system. Relays are exploited to improve performance in wireless communication systems. The advantages of the cooperative diversity $[4,7]$ protocols together with CSMA techniques come at the expense of a reduction in the spectral efficiency since the relays must transmit on orthogonal channels in order to avoid interfering between the source node and each other as well. Hence for a regular cooperative diversity network with $\mathrm{M}$ relaying nodes, $\mathrm{M}+1$ channels are employed, which incurs a bandwidth penalty. This problem of the inefficient use of the channel resources can be eliminated with the use of the best-relay selection scheme. In such a scheme, the best relay node only is selected to retransmit to the destination [4]. Hence, two channels only are required in this case (regardless of the number of relays). In this paper, we investigate the error rate performance in a multiple path amplify-and forward relay with best-relay selection network using orthogonal frequency division multiplexing (OFDM) Signals. Multiple-relay cooperative diversity in M-ary QAM OFDM-based systems with Best Relay Selection has better performance than the regular cooperative system.

OFDM is the modulation technique used in many new broadband communication schemes, including digital television, digital audio broadcasting, DSL/ADSL and wireless LANs. It also allows digital data to be efficiently and reliably transmitted over a radio channel, even in multipath environments [11-13]. In OFDM, although sub-carriers overlap, this does not create any problem since they are orthogonal, that is, the peak of one occurs when that of others are at zero. This is achieved by realizing all the subcarriers together using the inverse fast Fourier transform (IFFT). The analysis 


\section{9 \\ ISSN (Print) : 2320 - 3765 \\ ISSN (Online): $2278-8875$ \\ International Journal of Advanced Research in Electrical, Electronics and Instrumentation Engineering}

(An ISO 3297: 2007 Certified Organization)

\section{Vol. 4, Issue 2, February 2015}

of BER performances have suggested that OFDM is better than CDMA which is currently incorporated in most existing $3 \mathrm{G}$ systems $[4,5]$. A major problem in most wireless systems is the presence of a multipath channel. In this environment, the transmitted signal reflects off several objectives and a result, multiple delayed versions of the transmitted signal arrive at the receiver which causes the received signal to be distorted. Many wired systems also have a similar problem with reflection occurring due to impedance mismatches in the transmission line [10]. A multipath channel will cause two problems for an OFDM system. The first is ISI which occurs when the received OFDM symbol is distorted by the previously transmitted OFDM symbol and has a similar effect to the ISI that occurs in a singlecarrier system [12]. However, in such systems, the interference is typically due to several symbols other than only the previous ones; and the symbol period is typically much shorter than the time span of the channel, whereas the typical OFDM symbol period is much longer than the time span of the channel.

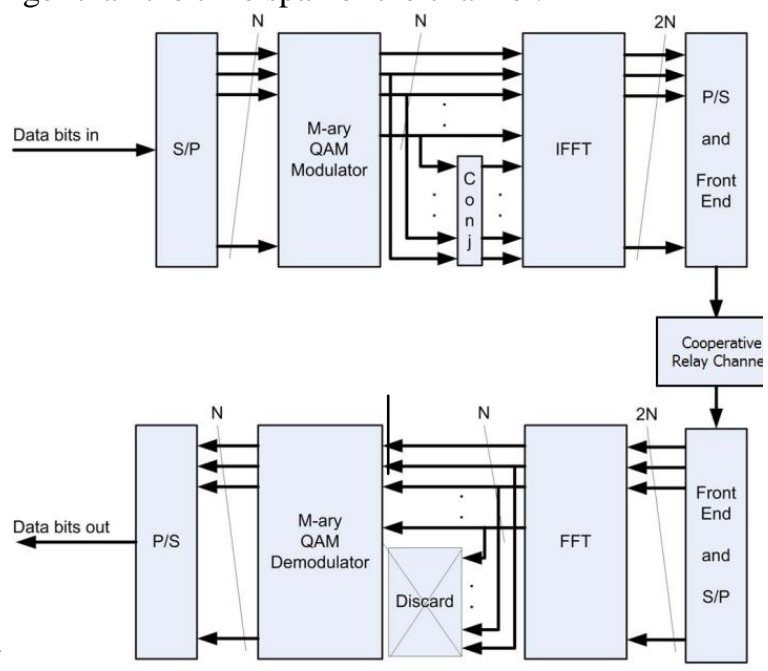

Fig. 1.2 Block diagram of M-ary QAM on OFDM Relay Network System.

\section{SYSTEM FUNCTIONING}

OFDM TRANSCEIVER : In this section, we describe the OFDM transceiver system. Before transmitting information bit over an AWGN channel through the OFDM transmitter, I use the M-PSK and M-QAM modulation schemes shown in Fig.1.2 The transmitter section converts the digital data to be transmitted, into a mapping of the sub-carrier's amplitude and phase using modulation tech

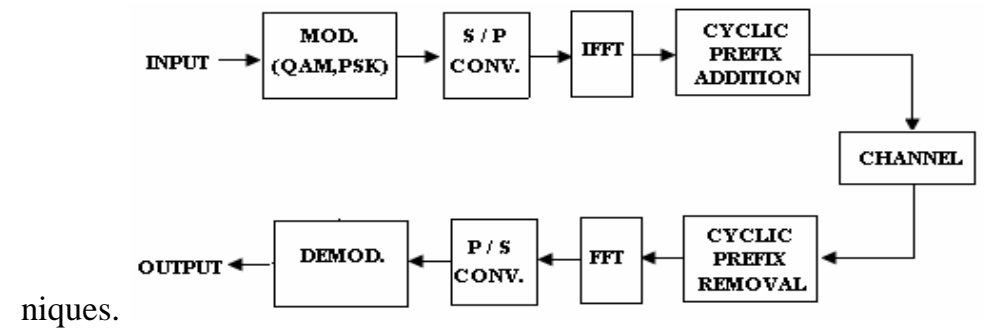

Fig. 1.3 Block diagram showing a basic OFDM transceiver

The spectral representation of the data is then transformed into the time domain using an IFFT which is much more computationally efficient and used in all practical Systems. The addition of a cyclic prefix to each symbol solves both ISI and inter-carrier interference (ICI). The digital data is then transmitted over the channel. After the time-domain signal passes through the channel, it is broken down into the parallel symbols and the prefix is simply discarded. The receiver performs the reverse operation to that of the transmitter. The amplitude and phase of the sub-carrier are then selected and converted back to digital data. In OFDM, multiple sinusoidals with frequency separations $1 / T$ are used, where $T$ is the active symbol period. The information to be sent on each sub-carrier $k$ is multiplied by its corresponding 


\section{9 \\ International Journal of Advanced Research in Electrical, Electronics and Instrumentation Engineering}

ISSN (Print) : $2320-3765$

ISSN (Online): $2278-8875$

(An ISO 3297: 2007 Certified Organization)

\section{Vol. 4, Issue 2, February 2015}

carrier $g_{k}(t)=e^{\frac{j 2 \pi k t}{T}}:$ and the sum of such modulated sinusoidals forms the transmit signal. Therefore, the sinusoidal used in OFDM can be defined as [12]

$$
g_{k}(t)=\frac{1}{\sqrt{T}} e^{\frac{j 2 \pi k t}{T}} w(t)
$$

where, $k=0,1, \ldots N-1$ corresponds to the frequency of the sinusoidal and $w(t)=u(t)-u(t-T)$ is a regular window over $[0$, $T]$. Since the OFDM system uses multiple sinusoidal signals with frequency separations of $1 / \mathrm{T}$, each sinusoidal is modulated by independent information. Mathematically we can write a transmit signal over the channel as,

$$
\begin{aligned}
S(t) & =\delta_{0} g_{0}(t)+\delta_{1} g_{1}(t)+\ldots+\delta_{N-1} g_{N-1}(t) \\
& =\sum_{0}^{N-1} \delta_{k} g_{k}(t) \\
& =\frac{1}{T} \sum_{0}^{N-1} \delta_{k} e^{\frac{j 2 \pi k t}{T}} w(t)
\end{aligned}
$$

Where $\delta_{k}$ is the $k$ th symbol in the message symbol sequence for $k$ in $[0, N-1]$, where $N$ is the number of carriers. $\mathrm{K}$ changes with bandwidth values.

\section{RELATIONSHIP BETWEEN ES/NO OR EB/NO FOR OFDM SYSTEM}

This section presents the relationship between the Es/N0 and Eb/N0 for the OFDM system. To conduct a Monte Carlo simulation of an OFDM system, the required amount of channel noise to be generated has to be representative of the required $E s / N O$ or $E b / N O$. In MATLAB it is easy to generate a Gaussian noise with a zero mean and unit variance which has to be scaled accordingly to represent the required $E b / N O$ or $E s / N O$. Normally, for a simple BPSK system[14], the bit energy and symbol energy are the same as its $E b / N O$ and Es/NO. However, if we use a MQAM or M-PSK modulation scheme for the OFDM system then they are not the same because each OFDM symbol contains an additional overhead in both the time and frequency domains [9,15]. In the time domain, the cyclic prefix is an additional overhead to each OFDM symbol being transmitted. In the frequency domain, not all the sub-carriers are utilized for transmitted the actual data bits, rather a few sub-carriers are unused and are reserved as guard bands. The relationship between the symbol energy and bit energy is [3]:

$$
\begin{aligned}
& \frac{E_{s}}{N_{0}}=\frac{E_{b}}{N_{0}}\left(\frac{n \cdot D S C}{n \cdot F F T}\right)\left(\frac{T_{d}}{T_{d}+T_{c p}}\right) \\
& \text { If we express the above equation in db, we can write } \\
& \frac{E_{s}}{N_{0}} d b=\frac{E_{b}}{N_{0}} d b+10 \log _{10}\left(\frac{n \cdot D S C}{n \cdot F F T}\right)+10 \log _{10}\left(\frac{T_{d}}{T_{d}+T_{c p}}\right)
\end{aligned}
$$

where, $T d$ is the data symbol duration, $T c p$ the cyclic prefix duration and $n . D S C$ the number of used subscribers used in the OFDM system.

\section{RELATION BETWEEN EB/NO AND ES/NO IN OFDM}

The relation between symbol energy and the bit energy is as follows:

$E s N 0=E b N 0$ (n.DSCn.FFT) TdTd $+T c p$

Expressing in decibels,

$E s N 0 d B=E b N 0 d B+10 \log 10(n \cdot D S C n \cdot F F T)+10 \log 10(T d(T d+T c p))$ 


\title{
(9) \\ International Journal of Advanced Research in Electrical, Electronics and Instrumentation Engineering
}

ISSN (Print) : $2320-3765$

ISSN (Online): 2278 - 8875

\author{
(An ISO 3297: 2007 Certified Organization)
}

\section{Vol. 4, Issue 2, February 2015}

\section{PAPER WORK AND OBJECTIVE}

Analysis \& study of the effects of filtering on the performance of a M-QAM modulation scheme on Random and fixed parameters of $\mathrm{x}$ input with $\mathrm{M}=4,8, \ldots, 512$ for the OFDM system then they are not the same because each OFDM symbol contains an additional overhead in both the time and frequency domains. In the time domain, the cyclic prefix is an additional overhead to each OFDM symbol being transmitted [16-18]. To overcome this and improve system performance, a simple effective method of Non coherent \& coherent approaches we use scatter plots of all these schemes. The above calculations are shared with a paper which is defined only with 256QAM but this paper is updated with 512-QAM with their Scatter graphs in broadband channel requirement in non coherent approach. The main objective of this paper is to develop a method based on transceivers that provides a simple but effective comparison between performance through M-ary QAM and splitting frames in bit error rate (BER) performance which is required in modern broadband wireless transmission OFDM systems[19].

\section{VI.SIMULATION RESULTS}

Simulation was performed to measure the BER in M- ary QAM OFDM scheme. We simulated BER versus SNR for Mary OFDM into 16 subchannels with the best-relay selection for numbers of relays (M) equal 3. All figures compares BER performance of M-ary QAM OFDM Bit based and with the best-relay selection scheme, showing the results only of 256-QAM OFDM and 512 QAM OFDM modulation plots or simulations. Scatter plots also produces the differences in results in terms of bth phases. At BER=10-5, 4QAM-16 will require a $E b / N o=6.9 \mathrm{~dB}$. 4QAM-16 with the bestrelay selection will require a $E b / N o=2.05 \mathrm{~dB}$. 16QAM-16 will require a $E b / N o=10.7 \mathrm{~dB}$. 16QAM-16 with the bestrelay selection will require a $E b / N o=6 \mathrm{~dB} .64 \mathrm{QAM}-16$ will require a $E b / N o=15 \mathrm{~dB}$. 64QAM-16 with the best-relay selection will require a $E b / N o=10.2 \mathrm{~dB}$. The cooperative diversity with best-relay selection provides a performance improvement of nearly $4.7 \mathrm{~dB}$.
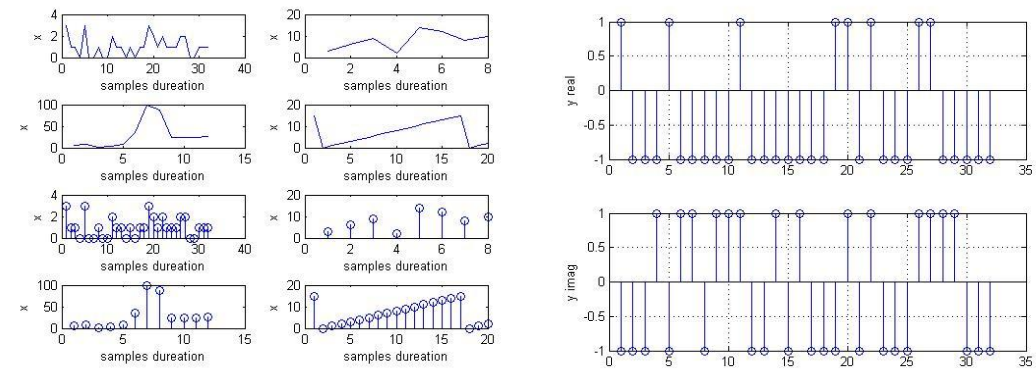

Fig. 6.1 Cumulative plot of 512 QAM-OFDMFig. 6.2 Data - mixing plot of 512 QAM-OFDM
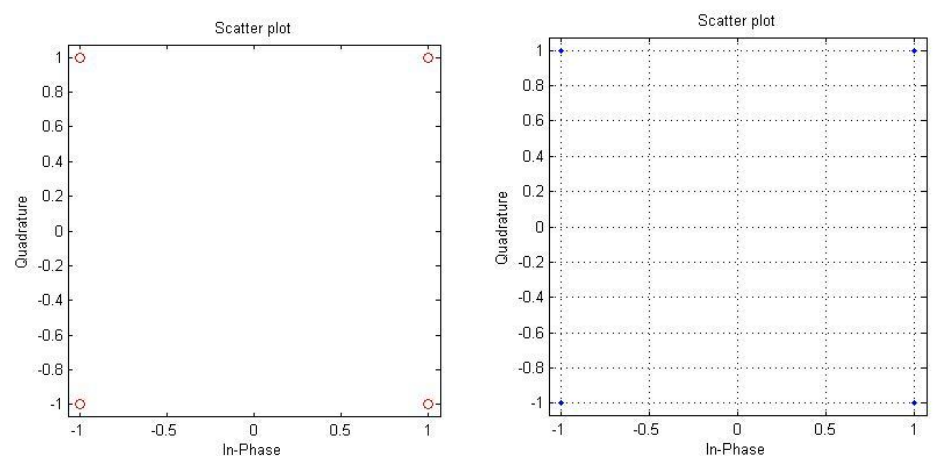

Fig. 6.3 Scatter plot of mixing Fig. 6.4 Scatter plot of mixing QAM 


\title{
(9) \\ International Journal of Advanced Research in Electrical, Electronics and Instrumentation Engineering
}

ISSN (Print) : $2320-3765$

ISSN (Online): $2278-8875$

\author{
(An ISO 3297: 2007 Certified Organization) \\ Vol. 4, Issue 2, February 2015

\section{CONCLUSION}

The results show M-ary QAM OFDM-based system with Best Relay Selection the best-relays selection cooperative has BER better than the regular cooperative diversity. It was found that the performance gain between Mary QAM OFDM-based system with Best Relay Selection the best-relays selection cooperative and the regular cooperative diversity (irrespective of modulation scheme used) was about $0.29-0.45 \mathrm{~dB}$. When we change modulation scheme for higher capacity, we can increase number of subchannel and use cooperative diversity with best-relay selection for higher performance. In this paper, we investigate only amplify and forward cooperative diversity system in the multiple path. In OFDM, although sub-carriers overlap, this does not create any problem since they are orthogonal, that is, the peak of one occurs when that of others are at zero [18]. This is achieved by realizing all the subcarriers together using the inverse fast Fourier transform (IFFT). The analysis of BER performances have suggested that OFDM is better than CDMA which is currently incorporated in most existing 3G systems $[4,5,19]$. The major problem is resolved by using 256 QAM OFDM scheme in most wireless systems is the presence of a multipath channel and broadband services of [19] TV transmission and mobile channel operations. In this environment, the transmitted signal reflects off several objectives and a result, multiple delayed versions of the transmitted signal arrive at the receiver which causes the received signal to be distorted.

\section{REFERENCES}

[1] J. Chuang and N. Sollenberger, "Beyond 3G: Wideband wireless data access based on OFDM and dynamic packet assignment," IEEE Communications Mag., vol. 38, pp. 78- 87, July 2000.

[2] Saltzberg, B. R., "Performance of an Efficient Parallel Data Transmission System," IEEE Trans. on Communications, Vol. COM-15, No. 6, December 1967, pp. 805-811, 2011.

[3] A.G.Armada, "Understanding the Effects of Phase Noise in OFDM," IEEE Transaction on Broadcasting, vol. 47, No.2, June 2012.

[4] Mehul Jain and M. Mani Roja, "Comparison of OFDM with CDMA System in Wireless telecommunication for multipath delay spread," The 1st IEEE and IFIP International Conference in Central Asia, 26-29 Sept., 2014 Pages 5.

[5] Bernard Sklar, Digital Communication Fundamentals and Applications, Second Edition, Pearson Education, Asia, 2010.

[6] Sami H. O. Salih, Mamoun M. A. Suliman, "Implementation of Adaptive Modulation and Coding Techniques using Matlab," 53rd International Symposium ELMAR-2011, 14-16 September 2011, Zadar, Croatia.

[7] Sanjeev Kumar, Swati Sharma, "Error Probability of Different Modulation Schemes for OFDM based WLAN standard IEEE 802.11a" International Journal of Engineering (IJE), Volume: 4, Issue: 4,2014.

[8] L. Hanzo, W. Webb, and T. Keller, "Single and Multi-carrier Quadrature Amplitude Modulation", New York, USA: IEEE Press-John Wiley, April 2000.

[9] S. B. Weinstein and P. M. Ebert, "Data transmission by frequency division multiplexing using the discrete Fourier transform," IEEE Transactions on Communication Technology, vol. COM-19, pp. 628-634, October 2014.

[10] X. Cai and G. B. Giannakis, "Low-complexity ICI suppression for OFDM over time- and frequency-selective Rayleigh fading channels," in Proc. Asilomar Conf. Signals, Systems and Computers, Nov. 2012.

[11] Shaoping Chen and Cuitao Zhu, "ICI and ISI Analysis and Mitigation for OFDM Systems with Insufficient Cyclic Prefix in Time-Varying Channels" IEEE Transactions on Consumer Electronics, Vol. 50, No. 1, February 2014.

[12] M. S. Islam, G. R. Barai, and A. Mahmood, "Performance analysis of different schemes using OFDM techniques in Rayleigh fading channel," International Journal of Fundamental Physics Science, Vol. 1, No. 1, pp. 22-27, June, 2011.

[13] van Wyk, J. and Linde, L., "Bit error probability for a M-ary QAM OFDM-based system," in IEEE Trans. On Wireless Comm., , pp. 1-5, 2007

[14] Salama S. Ikki and Mohamed H. Ahmed, "Performance of Multiple-Relay Cooperative Diversity Systems with Best Relay Selection over Rayleigh Fading Channels," in Hindawi Publishing Corporation EURASIP Journal on Advances in Signal Processing, vol. 2008, Article ID 580368, pp. 7, March 2008

[15] J. G. Proakis, Digital Communications, ch. 5-2-2, pp. 257-282,ch. 14-4-3, pp. 777-793. McGraw-Hill, 4th ed.,2014.

[16] D. Yoon, K. Cho, and J. Lee, "Bit error probability of M-ary quadrature amplitude modulation," in Proc. IEEE VTS-Fall VTC '00, (Boston, MA,USA), 24-28 September 2010.

[17] Yi Zhao, Raviraj Adve and Teng Joon Lim, "Symbol Error Rate of Selection Amplify-and-Forward Relay Systems," IEEE Trans. on Wireless Comm., vol. 10, no.11, pp. 757-759, November 2013.

[18] M. O.Hasna andM.-S. Alouini, "Harmonic mean and end-toend performance of transmission systems with relays," IEEE Transactions on Communications, vol. 52, no. 1, pp. 130-135, 2014.

[19] S. S. Ikki and M. H. Ahmed, "Performance analysis of cooperative diversity wireless networks over Nakagami-m fading channel," IEEE Communications Letters, vol. 11, no. 4, pp. 334-336, 2014.

[20] M. O.Hasna andM.-S. Alouini, "Harmonic mean and end-toend performance of transmission systems with relays," IEEE Transactions on Communications, vol. 52, no. 1, pp. 130-135, 2013.

[21] S. S. Ikki and M. H. Ahmed, "Performance analysis of cooperative diversity wireless networks over Nakagami-m fading channel," IEEE Communications Letters, vol. 11, no. 4, pp. 334-336, 2014. 


\title{
International Journal of Advanced Research in Electrical, Electronics and Instrumentation Engineering
}

\author{
(An ISO 3297: 2007 Certified Organization)
}

\section{Vol. 4, Issue 2, February 2015}

\section{BIOGRAPHY}
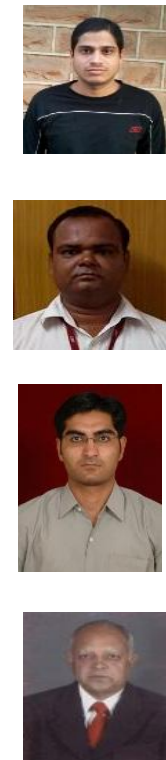

Ritesh Saraswat completed his B. E. (Electronics \& Comm. Engineering), K.L.S.G.I.T., Karnataka University Dharwad , 2001. He completed his M. E. (Digital Communication), M.B.M. Engg. College, JNVU (Jodhpur), 2007, and Ph.D. (Pursuing) from JNU, Jodhpur.

Vishwajeet Mishra Completed his B.E. Degree in Electronics \& Communication Engineering Branch from Rajathan University, Jaipur, Rajasthan, India in 2007, M.Tech. in Digital communication from JNU, Jodhpur, India in 2011

Piyush vyas completed his B.Sc. Degree in Electronics Engineering Branch from JNVU, Jodhpur, Rajasthan, India in 2004, M.Sc. in Electronics Engineering Branch from Devi Ahilya Vishwa Vidfhyalaya University, Indore, MP., India in 2006, and M.Tech. in Communication Engineering Branch from VIT University, Vellore, Tamilnadu, India in 2009. He is pursuing PhD from JNVU, Jodhpur, Rajashthan from 2013.

Prof. K. K. Arora Completed his B.E.\& M.E. Degree in Electronics Engineering Branch from MBM Engineering College, JNVU, Jodhpur, Rajasthan, India in Batch 1985, He is pursuing PhD. He has also completed $\mathrm{MBa}$ from KOU, Kota, Rajasthan, India. 